\title{
OSTEOID OSTEOMA AS AN ATYPICAL SOLITARY LUCENT EPIPHYSEAL LESION IN A CHILD
}

\author{
Slavica PONORAC*, Damjana KLJUČEVŠEK
}

Children's Hospital, University Medical Centre Ljubljana, Slovenia

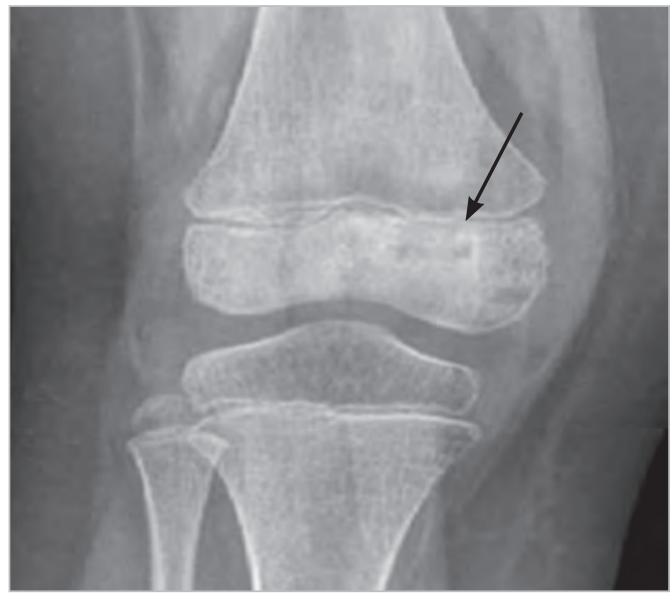

Fig. 1 Radiograph showed a subtle lytic lesion (arrow) with diffuse medullary sclerosis of the right medial femoral condyle epiphysis.
A previously healthy 5-year-old boy was admitted to the hospital with a nine-month history of diurnal and nocturnal pain in lower legs, particularly in the right knee, partially relieved by NSAIDs. Routine laboratory tests and an initial radiographic evaluation six months ago were normal. Due to persistent pain and limping repeated radiographs identified subtle lytic lesion with diffuse medullary sclerosis of the medial femoral condyle epiphysis (Fig1). MRI of the knee presented T2 hyperintense lesion with a thin hypointense rim consistent with sclerosis and perilesional bone marrow edema. After paramagnetic contrast application the lesion revealed a ring of enhancement (Fig. 2 a, b). A bone scan showed no focal uptake. Imaging char-

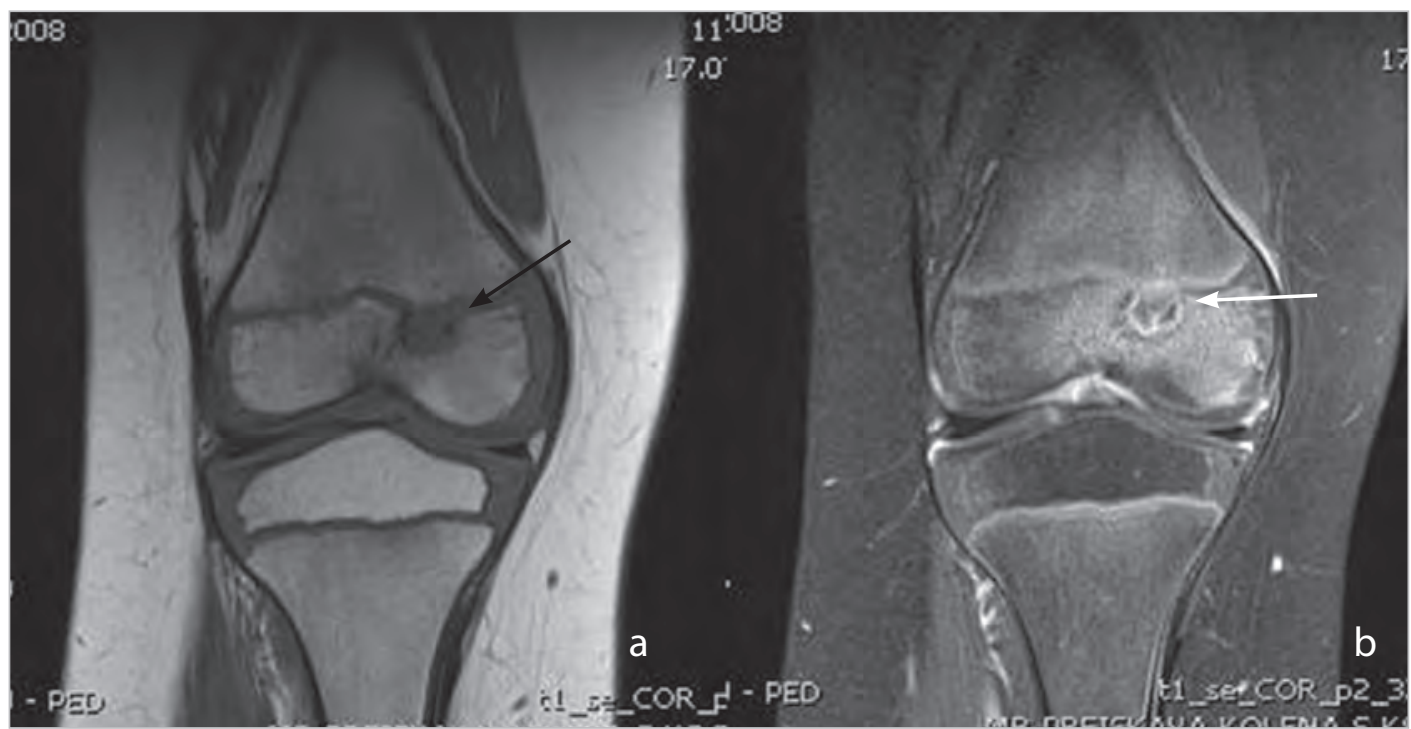

Fig. 2 MR images demonstrated a rounded T1 hypointense lesion with thin rim of sclerosis (arrow) and perilesional marrow edema. A ring of enhancement around the lesion (white arrow) was seen after application of contrast media. 


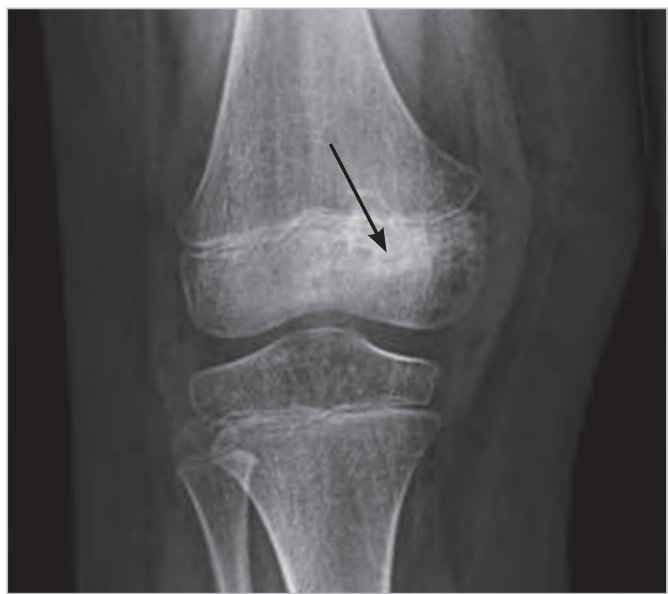

Fig. 3 Control radiograph after surgical excision showed a large area of reactive sclerosis (arrow).

acteristics (radiographs and MRI) pointed to subacute osteomyelitis (e.g. Brodie abscess) as a main differential diagnosis, however nocturnal pain was suggestive of an atypical osteoid osteoma. The bone biopsy was inconclusive. Following a complete surgical excision and histopathologic analysis of the lesion the diagnosis of osteoid osteoma was established. Control radiographs after surgery showed a healing epiphyseal lesion (Fig. 3). Several pathologic entities should be considered as possible etiologies when confronted with a solitary lytic epiphyseal lesion. Osteomyelitis is one of the commonest causes, along with chondroblastoma, which affects the epiphysis almost exclusively. Eosinophilic granuloma, enchondroma, tuberculosis, foreign body granuloma, and osteoid osteoma are described only in a few examples. In most cases, the classic appearance of an osteoid osteoma does not pose a diagnostic challenge. However, lesions displayed nonspecific clinical findings may share similar radiologic appearances or differ from their classical description. In our case none of the imaging modalities were able to make final diagnosis.

Key words: Osteoid osteoma - Lucent epiphyseal lesion - Imaging.

Authors' contributions: Conception and design: DK, SP; Acquisition, analysis and interpretation of data: DK, SP; Revising it critically for important intellectual content: DK, SP.

Conflict of interest: The authors declare that they have no conflict of interest.

*Corresponding author: slavi.ponorac@gmail.com Tel.: + 386152292 64; Fax.: + 38615222993

Received: May 12, 2015; Accepted: June 1, 2015 